Cochrane Database of Systematic Reviews

\title{
Pharmacological treatment for chronic central neuropathic pain in people with multiple sclerosis (Protocol)
}

James E, Young CA, Gibbons EL, Shueb A, Tur C

James E, Young CA, Gibbons EL, Shueb A, Tur C.

Pharmacological treatment for chronic central neuropathic pain in people with multiple sclerosis (Protocol).

Cochrane Database of Systematic Reviews 2020, Issue 4. Art. No.: CD013599.

DOI: 10.1002/14651858.CD013599.

\section{www.cochranelibrary.com}


TABLE OF CONTENTS

HEADER 1

ABSTRACT

BACKGROUND

OBJECTIVES

METHODS

ACKNOWLEDGEMENTS

REFERENCES

APPENDICES

HISTORY

CONTRIBUTIONS OF AUTHORS

DECLARATIONS OF INTEREST

SOURCES OF SUPPORT 
[Intervention Protocol]

\section{Pharmacological treatment for chronic central neuropathic pain in people with multiple sclerosis}

Eleanor James ${ }^{1}$, Carolyn A Young², Emily L Gibbons ${ }^{3}$, Akrem Shueb ${ }^{4}$, Carmen Tur 5

1Barts and The London School of Medicine and Dentistry, Queen Mary University of London, London, UK. 2The Walton Centre NHS Foundation Trust, Liverpool, UK. ${ }^{3}$ Department of Neurology, The Walton Centre NHS Foundation Trust, Liverpool, UK. ${ }^{4}$ Department of Psychiatry, Southern Hill Hospital, Norwich, UK. ${ }^{5}$ Department of Neuroinflammation, UCL Institute of Neurology, London, UK

Contact address: Carmen Tur, c.tur@ucl.ac.uk.

Editorial group: Cochrane Multiple Sclerosis and Rare Diseases of the CNS Group.

Publication status and date: New, published in Issue 4, 2020.

Citation: James E, Young CA, Gibbons EL, Shueb A, Tur C. Pharmacological treatment for chronic central neuropathic pain in people with multiple sclerosis (Protocol). Cochrane Database of Systematic Reviews 2020, Issue 4. Art. No.: CD013599. DOI: 10.1002/14651858.CD013599.

Copyright @ 2020 The Cochrane Collaboration. Published by John Wiley \& Sons, Ltd.

\section{A B S T R A C T}

\section{Objectives}

This is a protocol for a Cochrane Review (intervention). The objectives are as follows:

To assess the absolute and comparative benefit and tolerability of pharmacological therapies for the treatment of central neuropathic pain syndromes in people with MS. 


\section{B A C K G R O U N D}

Pain is a common symptom in people who have multiple sclerosis (MS). One systematic review of 17 studies, which included 5319 people, found an overall pain prevalence of $63 \%$ (95\% confidence interval (Cl) $55 \%$ to $70 \%$ ) (Foley 2013). Between $8 \%$ and $32 \%$ of people with MS regard pain as one of their most severe symptoms (Shibasaki 1974; Stenager 1991). Pain is associated with worse fatigue and sleep disturbance, and greater anxiety and depression in people with MS (Amtmann 2015). Health-related quality of life is adversely influenced by pain (Motl 2010; Yamout 2013).

\section{Description of the condition}

People with MS often have acute, paroxysmal, and chronic pains, some of which can co-exist in the same part of the body. Chronic pain is defined as persistent or recurrent pain lasting three months or longer according to the International Association for the Study of Pain (IASP) classification of chronic pain (www.iasp-pain.org).

The focus of this Cochrane Review will concern treatments for chronic central neuropathic pain. Central neuropathic pain arises as a direct consequence of a lesion or disease of the central somatosensory nervous system (Treede 2008; IASP; pain terms; www.iasp-pain.org/terminology).

People with MS who experience neuropathic pain typically explain dysaesthesia (i.e. an unpleasant abnormal sensation, whether spontaneous or evoked). This is mainly described as pins and needles, numbness, or a burning sensation in the affected area. Sometimes people experience hypersensitivity in the affected area. When this abnormal sensation is evoked by usually nonpainful stimuli, it is called allodynia. The term hyperaesthesia or hyperalgesia is reserved for cases where the abnormal sensation is evoked by generally painful stimuli, which, in this case, cause an exaggerated painful reaction (according to IASP).

The prevalence of chronic central neuropathic pain in people with MS increases with physical disability and duration of illness (O'Connor 2008). Correlation of pain prevalence with MS disease milestones, such as relapse, and over longitudinal follow-up has not been well-documented (Foley 2013). From the variations in description, intensity, neurophysiological findings, and response to therapy, it is clear that 'chronic central neuropathic pain' is a heterogeneous group of disorders. One study that classified central neuropathic pain in people with MS attending a clinic found that $86 \%$ had central pain that affected their lower limbs, trunk, or upper limbs and, for 14\%, trigeminal neuralgia only (Osterberg 2005). Trigeminal neuralgia in people with MS is increasingly believed to be due to brainstem demyelination and is therefore considered to be centrally induced (Cruccu 2016).

\section{Description of the intervention}

A range of drugs have been used for neuropathic pain, including tricyclics, selective noradrenaline reuptake inhibitors, opioids, alpha-2-delta ligands (e.g. gabapentin, pregabalin), sodium channel blockers, and other antiepileptic drugs. However, such prescription is largely based on small trials or from guidelines that extrapolate the evidence from peripheral non-MS neuropathic pain to MS (Pöllmann 2008; Attal 2010), although Chitsaz 2009 undertook a randomised open trial of nortriptyline in 59 people with MS. Cannabinoids have been investigated in randomised placebo-controlled trials with conflicting results (Svendsen 2004;
Rog 2005; Langford 2013). Trial data on invasive treatments, such as intrathecal drugs or spinal cord stimulation, for pain in people with MS are sparse. There is also limited evidence on complementary therapies. Overall, the pharmacological treatment of central neuropathic pain in people with MS is challenging and may require polypharmacy, causing more frequent drug interactions and increasing the risk of complications for the patient.

\section{How the intervention might work}

The pharmacological management of neuropathic pain has been based upon extrapolation of the effects of drugs, such as anticonvulsants in epilepsy suppressing aberrant nerve conduction, serendipity, and an increasing understanding of the pathophysiological mechanisms underlying neuropathic pain, which have been reviewed extensively (Besson 1999; Woolf 2000; Hansson 2001; Jensen 2001; Koltzenburg 2001; Baron 2006).

Painful sensations are usually conveyed following appropriate stimulation of unmyelinated (C) fibres, which synapse in the superficial laminae of the dorsal horn and thinly myelinated (Aס) fibres in deeper laminae. The second-order projection neuron is of the wide dynamic range type. It receives input from nociceptive ( $C / A \delta$ fibres) and non-nociceptive ( $A \beta$ fibres) inputs and both descending and gamma aminobutyric acid (GABA) interneuron inhibitory input, together with glial cells. Wide dynamic range cells are in part characterised by small receptive zones that can be excited by non-noxious stimuli, such as touch and gentle pressure, surrounded by a much larger zone from which noxious stimuli, pinch, firm pressure, or temperature can evoke neuronal discharges. These large receptive fields overlap, extending over several dermatomes, and their receptive fields reflect synaptic propriospinal interconnections in the dorsal horn that extend over several segments. After nerve injury the remaining peripheral and central neurons develop a combination of abnormal sensitisation and spontaneous ectopic activity.

Nerve lesions lead to the release of nerve growth factor and the expression of new receptors, for example the vanilloid (Hudson 2001) and adrenoceptors (Drummond 2014), upon remaining fibres. Sodium channel clusters also accumulate at both peripheral nerve lesion sites, but also proximally within the intact dorsal root ganglion (Hudson 2001). Two voltage-gated sodium channels, Nav1.8 and Nav1.9, are selectively expressed in nociceptive primary afferent neurons and an embryonic channel, Nav1.3, is upregulated in damaged peripheral nerves (Baron 2006). Spontaneous activity in C-fibres via several presynaptic (opioid receptors, calcium channels) and postsynaptic mechanisms (glutamate, noradrenaline, serotonin, and GABA receptors and sodium channels) are involved in the central sensitisation of second-order wide dynamic range neurons. This leads to light touch and punctate stimuli via mechanoreceptive $A \beta$-fibres being perceived as pain (dynamic and punctate mechanical allodynia). In addition, the descending and interneuron inhibitory systems acting upon the wide dynamic range neurons are dysfunctional, resulting in further central sensitisation. Peripheral nerve injury activates spinal cord glial cells, which further enhances excitability in wide dynamic range neurons by releasing cytokines and increasing glutamate levels. If A $\beta$ fibre input is blocked, allodynia disappears, but burning spontaneous pain persists, indicating that the latter probably is mediated by $\mathrm{C}$ fibres. Oscillations in the dorsal root ganglion membrane potential and ectopic firing occur via a combination of voltage-dependent, tetrodotoxin-sensitive sodium 
channels and a passive voltage-independent potassium leakage. In rodents, centrally sensitised neurons have also been demonstrated in the thalamus and somatosensory cortex after peripheral nerve injury (Guilbaud 1992).

These observations on the pathophysiological mechanisms of neuropathic pain increase understanding of the likely mode of action of the common treatments. Tricyclic antidepressants may relieve pain by sodium channel blockade (Wang 2004), whereas serotonin-noradrenaline reuptake inhibitors such as duloxetine cause a balanced inhibition of serotonin and noradrenaline (Baldessarini 2001). Antiepileptic drugs may be useful in treating neuropathic pain because of their effects on voltage-gated sodium or calcium channels. Opioids mimic the actions of endogenous opioids to activate $\mu$ opioid and other receptors (Pasternak 2010).

\section{Why it is important to do this review}

People with MS often report low satisfaction with pain management (Solaro 2013). Management pathways may seem disorganised, efficacy inadequate, and people may have concerns about adverse effects. Yet, clinicians seeking comprehensive advice on best prescribing for chronic neuropathic pain in people with MS will find that there is a single systematic review that covers neuropathic pain that is not related to spasticity or trigeminal neuralgia (Jawahar 2013). Based on comprehensive searches and transparent assessment of study methodology, our systematic review will aim to provide assessment of the eligible primary studies of pharmacological treatments for central pain syndromes in people with MS, and summarise their benefit and tolerability.

\section{O B JE C T IVES}

To assess the absolute and comparative benefit and tolerability of pharmacological therapies for the treatment of central neuropathic pain syndromes in people with MS.

\section{METHODS}

\section{Criteria for considering studies for this review}

\section{Types of studies}

We will include double-blind, randomised controlled trials (RCTs) of treatment duration longer than seven days, which are either placebo-controlled or compare two or more agents. We will exclude quasi-randomised, non-randomised, and open trials. We will include cross-over trials with a minimum length of washout period of one week and we will contact the study authors to obtain information about the results of each period of the study. All trials with washout periods shorter than one week will be excluded. We will not exclude studies based upon reported outcomes. Only those studies published as full-text will be included.

\section{Types of participants}

We will include adults (aged over 18 years) with a clinically definite diagnosis of MS, using diagnostic criteria applicable at time of study design (Poser 1983; McDonald 2001; Polman 2005; Polman 2011; Thompson 2018), and at any stage of their disease. Types of chronic central pain syndromes included will be dysaesthetic extremity pain, painful paroxysmal symptoms (e.g. Lhermitte's phenomenon), and trigeminal neuralgia. Both spontaneous and evoked types of chronic central pain syndromes will be included. If evoked, both hyperalgesia (increased response to a painful stimulus) and allodynia (a painful response to a normally nonpainful stimulus) will be included.

We will exclude people who are recruited to a study within one month of a relapse. We will exclude studies that include people with other diagnoses unless we can obtain individual data for the participants with MS either from the published results or through contact with the study authors.

\section{Types of interventions}

We will include trials if they compare one active treatment with placebo or another active treatment in people with MS experiencing chronic central pain syndromes. Specific drug types that we will include will be: anticonvulsants, antidepressants, antispasmodics, opiates, non-steroidal anti-inflammatory drugs (NSAIDs), local anaesthetic and membrane-stabilising agents, steroids, and other classes of analgesics shown to be useful in other neuropathic pain conditions, such as capsaicin. We will not include cannabinoids that are considered in another Cochrane protocol (Filippini 2019).

We will pay attention to active comparator interventions (e.g. a different variant of the same drug, a different drug, or a nonpharmacological comparator). All the potential comparisons will be carefully specified. We will also indicate specific aspects related to the intervention or comparator such as route of administration, duration of intervention, and frequency. We will include comedications if the medications added to the main investigation drug were used in all the comparison groups.

\section{Types of outcome measures}

\section{Primary outcomes}

Benefit

- Percentage of participants reporting at least $50 \%$ improvement in pain intensity at end of treatment period compared to baseline (Moore 2010). Acceptable measures will be composite neuropathic pain score, as the McGill Pain Questionnaire (MPQ) (Melzack 1975), Short Form McGill Pain Questionnaire (SFMPQ) (Melzack 1987), Galer Neuropathic Pain Scale (Galer 1997), Numeric Rating Scale-Pain Intensity (NRS-PI) (Ferreira-Valente 2011), or the visual analogue scale (VAS) (Carlsson 1983).

- Patient Global Impression of Change (PGIC): number of participants reporting much or very much improvement in the PGIC. Please note that PGIC provides a patient-reported assessment of overall change in health status and it is not a measure of pain.

- Clinical Global Impression of Change (CGIC): number of participants reporting much or very much improvement in the CGIC.

\section{Safety}

- Incidence of all adverse events, including specific central nervous system adverse effects such as cognitive impairment, drowsiness, ataxia, and confusion.

- Number of participants with at least one serious adverse event. A serious adverse event will be considered if the outcome of the adverse event is death, or a life-threatening condition, or a condition that requires hospitalisation. The development of a clinical MS relapse will also be considered as a serious adverse event. 
- Number of participants withdrawing from the study due to adverse events or reducing dose due to adverse events.

\section{Secondary outcomes}

- Quality of Life measured by the McGill Quality of Life Questionnaire (Cohen 1995), Short Form-36 Health Survey (SF-36) (Ware 1992), and Multiple Sclerosis Quality of Life-54 (MSQOL-54) (Vickrey 1995).

- Percentage of participants reporting at least 30\% improvement in pain intensity at end of treatment period compared to baseline (Moore 2010).

\section{Search methods for identification of studies}

We will apply no language restrictions to the searches.

\section{Electronic searches}

The Information Specialist will search the Cochrane Multiple Sclerosis and Rare Diseases of the CNS Group Trials Register which, among other sources, contains trials from the following sources.

- Cochrane Central Register of Controlled Trials (CENTRAL) (latest issue).

- MEDLINE (PubMed) (1966 to date).

- Embase (1974 to date).

- CINAHL (EBSCOhost) (1981 to date).

- LILACS (Bireme) (1982 to date).

- PEDro (1990 to date).

- ClinicalTrials.gov (www.clinicaltrials.gov).

- World Health Organization (WHO) International Clinical Trials Registry Platform (ICTRP) (apps.who.int/trialsearch).

Information on the Group's Trials Register and details of search strategies used to identify trials can be found in the 'Specialised Register' section within the Cochrane Multiple Sclerosis and Rare Diseases of the Central Nervous System Group's website (msrdcns.cochrane.org/). The keywords used to search for trials for this review are listed in Appendix 1.

In conjunction with the Group's Information Specialist, we will search regulatory data from the European Medicines Agency (www.ema.europa.eu/ema/) and the Food and Drugs Administration (www.fda.gov/Drugs/InformationOnDrugs/).

We will not perform a separate search for adverse effects of the drugs included in the review. We will consider adverse effects described in included studies only.

\section{Searching other resources}

We will perform the following:

- check the reference lists from published reviews on symptom control in MS and identified RCTs;

- contact drug manufacturers for drugs identified in relevant RCTs;

- check the UK Clinical Research Network Study Portfolio (www.sheffield.ac.uk/library/cdfiles/ukcrn), including the Medical Research Council Clinical Trials Directory (www.ctu.mrc.ac.uk/);
- identify unpublished trials by contacting drug manufacturers and checking the UK Clinical Research Network Study Portfolio and the Medical Research Council Clinical Trials Directory;

- examine any relevant retraction statements and errata for included studies.

\section{Data collection and analysis}

\section{Selection of studies}

The review authors (EJ, CAY, EG, AS, and CT) will independently screen the titles and abstracts of papers identified using the search strategies to find studies that meet the predefined inclusion criteria. We will obtain the full-text articles of any potentially relevant articles. Afterwards, the same review authors will independently screen the full-text and identify studies for inclusion, and identify and record reasons for exclusion of ineligible studies. We will reach agreement by consensus after we assess the full-text article and we will contact the study authors for further information if necessary. We will list all studies excluded after fulltext assessment and their reasons for exclusion in a 'Characteristics of excluded studies' table. We will illustrate the study selection process in a PRISMA diagram.

Where studies have multiple publications, we will collate the reports of the same study so that each study, rather than each report, is the unit of interest for the review, and such studies have a single identifier with multiple references.

\section{Data extraction and management}

Three review authors (EJ, EG, and AS) will independently extract the participant and study characteristics (including dose regimens, trial duration, and outcome measures) and the outcomes data (as defined in Primary outcomes; Secondary outcomes) using a data collection form. The form will be piloted within the review team using three of the included studies, in order to ensure its usability. We will resolve any disagreements by discussion and consensus between all review authors.

In the data collection form, we will include the following study characteristics and list them in the 'Characteristics of included studies' table.

- Methods: study design, total duration of study, details of any 'run in' period (if applicable), number of study centres and location, study setting, and date of study.

- Participants: number of participants randomised, number of participants lost to follow-up/withdrawn, number of participants analysed, mean age, age range, gender, severity of condition, diagnostic criteria, trial duration, inclusion and exclusion criteria.

- Interventions: type of intervention, including drugs used and dose regimens, concomitant medications, and excluded medications.

- Outcomes: outcomes specified and collected, and time points reported.

- Notes: funding for trial, and notable conflicts of interest of trial authors.

One of the review authors (CT) will transfer data into Review Manager 5 (Review Manager 2014). Three review authors (EJ, EG, and AS) will double-check that data are entered correctly by 
checking the data noted on the data collection form match those on the publications and by comparing the data presented in the systematic review with the data extraction form.

\section{Assessment of risk of bias in included studies}

The methodological criteria will be based on the Cochrane Handbook for Systematic Reviews of Interventions (Page 2019). Two review authors (EJ, CAY) will independently evaluate the methodological quality of the studies using the Cochrane 'Risk of bias' tool. For parallel-group RCTs, we will assess the key domains of sequence generation, allocation concealment, blinding, incomplete outcome data, selective outcome, and other biases. For cross-over trials, we will use the criteria outlined in the Cochrane Handbook for Systematic Reviews of Interventions (Higgins 2019a). The key domains assessed for bias in the crossover trials will be essentially the same as for the parallel-group trials, although special attention will be paid to carry-over effect. We will summarise the risk of bias judgements across different studies for each outcome included in the 'Summary of findings' tables.

We will judge a study at low risk of bias only if we rate all key domains at low risk of bias; if we rate one or more of the domains at high risk of bias, then we will deem that study at high risk of bias; if we assess one or more of the domains at unclear risk of bias, we will consider the study at unclear risk of bias. Also, we will assess the methodological quality of the studies and will pay particular attention to:

- whether participants were truly randomised to the study groups;

- whether participants, healthcare team, and pain assessors were blinded to the assigned therapy;

- whether the groups were truly identical in terms of stage of disease and pretreatment central neuropathic pain level;

- progress of disease during the trial (e.g. number of relapses);

- other concomitant drug treatments or non-drug treatments for pain (e.g. transcutaneous electrical nerve stimulation (TENS));

- assessment of pain; and

- adverse effects.

We will discuss any disagreements between the review authors on the methodological quality of the identified studies and will resolve these by consensus.

\section{Measures of treatment effect}

For binary outcomes, we will use odds ratios (ORs) for parallel and cross-over trials. We will attempt to analyse paired data from cross-over trials given that chronic central neuropathic pain do not resolve over time in people with MS. For the continuous outcome measures, where studies used the same scales, we will consider using mean differences (MDs). If different scales are used in the included studies, we will use standardised mean differences (SMDs). We will try and re-express the SMDs as the units of a familiar outcome scale. For all these measures, we will estimate the $95 \% \mathrm{Cls}$.

\section{Unit of analysis issues}

We will only include trials where the participants are individually randomised to therapeutic interventions. We will consider trials where participants undergo only one intervention (i.e. parallel design) or more than one (i.e. cross-over design), and, if appropriate, we will combine data from both trial types. Although MS is a neurodegenerative condition, we will include cross-over trials on the grounds that chronic neuropathic pain in MS tends to be relatively stable over time when it appears, without showing a progressive nature, at least in the short to medium term (i.e. within three to twelve months from onset).

For trials with a cross-over design, if data from the two periods are available, we will analyse, in principle, both periods of the trial. However, we will assess the risk of a carry-over effect individually for each cross-over trial. In particular we will carefully evaluate:

- trials with drugs that have a long excretion phase;

- trials with drugs that have known lasting effects; and

- trials with short washout periods (i.e. only trials with a minimum acceptable length of washout period of one week will be included in the review_.

If the risk of carry-over of a cross-over trial is high (i.e. one of the three above mentioned scenarios applies, and trial data are available), we may consider only the analysis of unpaired data from the first period of the trial, although the power of this analysis will be much lower than that initially thought by the study authors. If the risk of carry-over of a cross-over trial is high, but instead data are not available (only the results), we may consider excluding the trial.

In cross-over trials, we will also evaluate other aspects individually for each trial, such as the percentage of participants who drop out after the first period of the trial or the presence of a period effect. We will exclude cross-over trials where all participants receive treatment options synchronically, where it may be difficult to distinguish between treatment effects and disease-related outcome trends over time, and where potential analyses of unpaired data are not possible.

Trials that include more than two comparison groups will be included if they meet the inclusion criteria. In those cases, all possible comparisons will be carefully defined. Priority will be given to pair-wise comparisons between the placebo and the active arms (as if the different active arms belonged to different, independent trials).

\section{Dealing with missing data}

We will contact study authors to gather missing data. In particular, we will take missing data related to missing outcomes, missing summary data, and missing individuals (trial dropouts) especially into consideration. We will only consider trials where intentionto-treat analysis is either possible, if trial data are available, or reported, for meta-analysis. In case of between-trial heterogeneity, we will investigate (through sensitivity analyses) whether this can be explained by differences in the amount of missing data between the trials.

\section{Assessment of heterogeneity}

We will assess heterogeneity visually using forest plots, and we will look at the treatment effect and the degree of overlapping of the Cls for the treatment effect of all included trials.

In addition, we will perform $\mathrm{Chi}^{2}$ tests, under the assumption that under the null hypothesis of all individual treatment effects (of $k$ studies) being the same, $Q$ (i.e. the sum of the weighted squared 
differences between $k$ individual treatment effects and overall treatment effect) follows a $\mathrm{Chi}^{2}$ distribution with $k-1$ degrees of freedom. We will determine the weights assigned to each squared difference as the inverse of the variance of the treatment effect for each study. Whenever the $\mathrm{P}$ value for the $\mathrm{Chi}^{2}$ test is below 0.05 , we will assume there is a significant heterogeneity in treatment effects. We will also consider there is risk of heterogeneity when $P$ values are below 0.1, given the low power $\mathrm{Chi}^{2}$ tests have when individual studies have small sample sizes (Greenland 2008).

When we suspect heterogeneity, we will determine the $I^{2}$ statistic value as the ratio between $Q$ minus the degrees of freedom ( $k$ $1)$ and $Q(\times 100 \%)$. This will allow us to quantify the amount of heterogeneity across included trials (Greenland 2008).

If we find heterogeneity across trials, we will investigate the reasons behind it (i.e. clinical diversity, methodological diversity, or both). When the main source of heterogeneity is methodological diversity, we will undertake sensitivity analyses by repeating the calculation omitting the trials which have high risk of bias.

Should heterogeneity persist after we exclude studies that are at high risk of bias, we will analyse the reasons behind this heterogeneity within the remaining studies. If there is no heterogeneity after we exclude those studies that are at high risk of bias, we will analyse each study that is at high risk of bias separately. If needed, we will undertake further sensitivity analyses by reincluding each one of these studies individually.

When the main source of heterogeneity is clinical diversity, we will assess the relationship between participants' clinical characteristics, for example level of disability or clinical phenotype, and the treatment effect.

\section{Assessment of reporting biases}

If we identify at least 10 eligible studies for inclusion in the review, we will create and analyse a funnel plot to assess the potential existence of small-study bias. We will assess publication bias according to the recommendations on testing for funnel plot asymmetry (Sterne 2011), as described in the Cochrane Handbook for Systematic Reviews of Interventions (Page 2019).

\section{Data synthesis}

We will perform a random-effects meta-analysis, since we will assume that the included studies are not all estimating the same intervention effect but instead are estimating intervention effects that follow a distribution across studies (DerSimonian 1986).

Random-effects meta-analyses assume individual treatment effects follow a normal distribution with a mean equal to the overall treatment effect and a given variance which depends directly on the differences between treatment effects and inversely on the weights of each trial. We will compute the weights of the studies as the inverse of the variance of the treatment effect. The use of a random-effects meta-analysis will not substitute the assessment of the source of heterogeneity, explained above.

For continuous outcomes, we will combine the results of parallel and cross-over trials through obtaining an MD (or SMD) between treatment arms using a random-effects model (Curtin 2002a). For dichotomous outcomes, we will combine ORs with $95 \% \mathrm{Cls}$ from parallel and cross-over trials according to the method of Curtin et al. (Curtin 2002b). In this method, the marginal OR estimates from cross-over and parallel trials will be pooled using a randomeffects model to estimate a weighted average of the OR. For adverse events, similar strategies as described above will be used, for continuous and binary outcomes, respectively.

If the review includes more than one comparison, for example if we have more than one effect measure which we cannot include in the same analysis, we will report the results for each effect measure separately.

Whenever possible, we will avoid the exclusion of trials based on their results and the presence of heterogeneity. Should we find outlying studies, we will perform analyses and estimate summary effect measures with and without them, as part of the sensitivity analyses. We will consider excluding these outlying studies if there is an unclear risk of bias. In addition, we will consider excluding other trials, especially cross-over trials, if there is risk of bias or if this is unclear. For cross-over trials, we will exclude studies with clear risk of carry-over effects (unless there are available unpaired data from first period to be analysed) or when all participants received treatment interventions synchronically, as explained above.

We will conduct analyses using Review Manager 5 (Review Manager 2014).

We will conduct the review according to this published protocol and report any deviations from it in the 'Differences between protocol and review' section of the systematic review.

\section{Subgroup analysis and investigation of heterogeneity}

Whenever we find statistical heterogeneity of which the source is clinical diversity across included studies, thus suggesting a genuinely different treatment effect in different clinical subgroups, we will consider (clinical) subgroup analyses, which we will mainly determine by the results of the assessment of the source of heterogeneity. Additionally, we will perform subgroup analyses to explore the presence of different treatment effects based on clinical characteristics, such as level of disability based on the Expanded Disability Status Scale (EDSS) score (Kurtzke 1983), or clinical phenotype. A possible reason why the level of disability or the clinical phenotype could be a source of heterogeneity may be related to the cause of the neuropathic pain, which can be associated to inflammatory factors (i.e. clinically eloquent inflammatory lesions in people with relatively early relapse-onset MS with lower EDSS scores), and associated to axonal damage or neurodegeneration in people with progressive MS, which generally have higher EDSS scores. The presence of concomitant treatment, which typically depends on the clinical phenotype, may also alter the effect of drugs for neuropathic pain in people with MS. In sum, the main two subgroups that will be compared are:

- people with relapsing-remitting MS, who usually have EDSS scores equal or below 4.0;

- people with (secondary or primary) progressive MS, who usually have EDSS scores above 4.0.

We will restrict subgroup analyses to outcomes that have a sufficient number of studies available. We will consider the relevance of subgroups where at least 10 studies for a subgroup analysis are available. We will interpret the results with caution. 


\section{Sensitivity analysis}

Whenever we find heterogeneity across included studies due to methodological diversity, we will identify the studies that lead to heterogeneity by undertaking sensitivity analyses. These will consist of the repetition of the estimation of the overall treatment effect omitting those trials at high risk of bias. Should heterogeneity persist after we exclude these trials, we may perform further sensitivity analyses by omitting first those studies with more extreme treatment effects (i.e. outlying trials). We will consider the exclusion of these outlying studies if there is an unclear risk of bias.

Independently of the presence of heterogeneity, we will use sensitivity analyses to assess the impact of studies of high risk of bias.

Additionally, we will perform sensitivity analyses to assess the impact of studies with missing data. Thus, these sensitivity analyses will estimate the overall treatment effect with and without considering trials with missing data. Whenever the impact of those trials with missing data is high, that is, when the overall conclusion on treatment effects change direction after omitting such trials with missing data, we will consider their exclusion from our analyses. This may indicate that the observed missing data are not missing at random. Importantly, if missing data are imbalanced between treatment arms, then this could affect harms and benefits differently. Therefore, in those studies that have missing data, we will analyse carefully the impact of missing data on the benefits and harms of the treatment being tested. Furthermore, whenever we have access to the original data of those trials with missing data, we will perform 'scenario analyses', where we will explore best-case, worst-case, and likely scenarios for the missing data. We will also mention the potential impact of missing data on our findings of the review in the 'Discussion' section.

\section{'Summary of findings' tables}

In the 'Summary of findings' tables, we will include an overall assessment of the evidence for the following outcomes.

- Percentage of participants reporting at least $50 \%$ improvement in pain intensity at end of treatment period compared to baseline.

- Number of participants reporting much or very much improvement in the PGIC.
- Number of participants reporting much or very much improvement in CGIC.

- Incidence of all adverse events.

- Number of participants with at least one serious adverse event.

- Number of participants withdrawing from the study due to adverse events or reducing dose due to adverse events.

- Quality of life scores from study baseline to end of treatment period.

We will produce two 'Summary of findings' tables with one comparison per table:

- active treatment versus placebo;

- one active treatment versus another active treatment.

In the 'Summary of findings' tables, we will give priority to longterm outcomes (i.e. six to twelve-month outcomes) if they are be available. Otherwise, we will include short-term outcomes (threemonth outcomes or even outcomes at shorter terms).

Two review authors (CT and CAY) will independently judgements the certainty of the evidence, with disagreements resolved by discussion or involving a third author (EJ). Judgements will be justified, documented, and incorporated into reporting of results for each outcome.

We will use the five GRADE considerations (risk of bias, consistency of effect, imprecision, indirectness, and publication bias) to assess the certainty of a body of evidence as it relates to the studies that contribute data to the meta-analyses for the prespecified outcomes. We will use methods and recommendations described in the Cochrane Handbook for Systematic Reviews of Interventions (Schünemann 2019), and will use GRADEpro software (GRADEpro GDT 2015). We will justify all decisions to downgrade or upgrade the certainty of the evidence using footnotes and we will make comments to aid the reader's understanding of the GRADE assessments where necessary.

\section{ACK N O WLEDGEMENTS}

We thank Prof Tim Friede for his contribution to designing the statistical analysis plan. We thank Dr David Rog for his assistance with drafting the protocol. 


\section{R E F E E N CE S}

\section{Additional references}

\section{Amtmann 2015}

Amtmann D, Askew RL, Kim J, Chung H, Ehde DM, Bombardier $\mathrm{CH}$, et al. Pain affects depression through anxiety, fatigue, and sleep in multiple sclerosis. Rehabilitation psychology 2015; 60(1):81-90.

\section{Attal 2010}

Attal N, Cruccu G, Baron R, Haanpää M, Hansson P, Jensen TS, et al. EFNS guidelines on the pharmacological treatment of neuropathic pain: 2010 revision. European journal of neurology 2010; 17(9):1113-e88.

\section{Baldessarini 2001}

Baldessarini RJ. Drugs for the treatment of psychiatric disorders. In: Hardman JG, Limbird LE, Gilman AG, editors(s). The pharmacological basis of therapeutics. 10th edition. New York (NY): McGraw Hill, 2001.

\section{Baron 2006}

Baron R. Mechanisms of disease: neuropathic pain - a clinical perspective. Nature clinical practice. Neurology 2006; 2(2):95-106.

\section{Besson 1999}

Besson JM. The neurobiology of pain. Lancet 1999; 353(9164):1610-5.

\section{Carlsson 1983}

Carlsson AM. Assessment of chronic pain I. Aspects of the reliability and validity of the visual analogue scale. Pain 1983; 16(1):87-101.

\section{Chitsaz 2009}

Chitsaz A, Janghorbani M, Shaygannejad V, Ashtari F, Heshmatipour M, Freeman J. Sensory complaints of the upper extremities in multiple sclerosis: relative efficacy of nortriptyline and transcutaneous electrical nerve stimulation. Clinical journal of pain 2009; 25(4):281-5.

\section{Cohen 1995}

Cohen SR, Mount BM, Strobel MG, Bui F. The McGill Quality of Life Questionnaire: a measure of quality of life appropriate for people with advanced disease; a preliminary study of validity and acceptability. Palliative medicine 1995; 9(3):207-19.

\section{Cruccu 2016}

Cruccu G, Finnerup NB, Jensen TS, Scholz J, Sindou M, Svensson $P$, et al. Trigeminal neuralgia: new classification and diagnostic grading for practice and research. Neurology 2016; 87(2):220-8. [DOI: 10.1212/WNL.0000000000002840] [PMID: 27306631]

\section{Curtin 2002a}

Curtin F, Altman D, Elbourne D. Meta-analysis combining parallel and cross-over clinical trials. I: Continuous outcomes. Statistics in medicine 2002; 21(15):2131-44.

\section{Curtin 2002b}

Curtin F, Elbourne D, Altman DG. Meta-analysis combining parallel and cross-over clinical trials. II: Binary outcomes. Statistics in medicine 2002; 21(15):2145-59.

\section{DerSimonian 1986}

DerSimonian R, Laird N. Meta-analysis in clinical trials. Controlled clinical trials 1986; 7(3):177-88. [PMID: 3802833]

\section{Drummond 2014}

Drummond PD. Neuronal changes resulting in up-regulation of alpha-1 adrenoceptors after peripheral nerve injury. Neural regeneration research 2014; 9(14):1337-40.

\section{Ferreira-Valente 2011}

Ferreira-Valente MA, Pais-Ribeiro JL, Jensen MP. Validity of four pain intensity rating scales. Pain 2011; 152(10):2399-404. [DOI: doi:10.1016/j.pain.2011.07.005]

\section{Filippini 2019}

Filippini G, Lasserson TJ, Dwan K, D'Amico R, Borrelli F, Izzo AA, et al. Cannabis and cannabinoids for people with multiple sclerosis. Cochrane Database of Systematic Reviews 2019, Issue 10. [DOI: $10.1002 / 14651858]$

\section{Foley 2013}

Foley PL, Vesterinen HM, Laird BJ, Sena ES, Colvin LA, Chandran S, et al. Prevalence and natural history of pain in adults with multiple sclerosis: systematic review and metaanalysis. Pain 2013; 154(5):632-42.

\section{Galer 1997}

Galer BS, Jensen MP. Development and preliminary validation of a pain measure specific to neuropathic pain: the Neuropathic Pain Scale. Neurology 1997; 48(2):332-8.

\section{GRADEpro GDT 2015 [Computer program]}

McMaster University (developed by Evidence Prime) GRADEpro GDT. Version accessed 20 January 2018. Hamilton (ON): McMaster University (developed by Evidence Prime), 2015.Available at gradepro.org.

\section{Greenland 2008}

Greenland S, O'Rourke K. Meta-analysis. In: Rothman KJ, Greenland S, Lash T, editors(s). Modern epidemiology. Philadelphia (PA): Lippincott Williams and Wilkins, 2008.

\section{Guilbaud 1992}

Guilbaud G, Benoist JM, Levante A, Gautron M, Willer JC. Primary somatosensory cortex in rats with pain-related behaviours due to a peripheral mononeuropathy after moderate ligation of one sciatic nerve: neuronal responsivity to somatic stimulation. Experimental brain research 1992; 92(2):227-45.

\section{Hansson 2001}

Hansson P, Lacerenza M, Marchettini P. Aspects of clinical and experimental neuropathic pain: the clinical perspective. In: Hansson P, Fields HL, Hill R, Marchettini P, editors(s). 
Neuropathic pain: pathophysiology and treatment. Seattle (WA): IASP Press, 2001:1-18.

\section{Higgins 2019a}

Higgins JP, Eldridge S, Li T. Chapter 23: Including variants on randomized trials. In: Higgins JP, Thomas J, Chandler J, Cumpston M, Li T, Page MJ, et al, editor(s). Cochrane Handbook for Systematic Reviews of Interventions version 6.0 (updated July 2019). Cochrane, 2019. Available from www.training.cochrane.org/handbook.

\section{Hudson 2001}

Hudson LJ, Bevan S, Wotherspoon G, Gentry C, Fox A, Winter J. VR1 protein expression increases in undamaged DRG neurons after partial nerve injury. European journal of neuroscience 2001; 13(11):2105-14.

\section{Jawahar 2013}

Jawahar R, Oh U, Yang S, Lapane KL. A systematic review of pharmacological pain management in multiple sclerosis. Drugs 2013; 73(15):1711-22.

\section{Jensen 2001}

Jensen TS, Gottrup H, Kasch H, Nikolajsen L, Terkelsen AJ, Witting N. Has basic research contributed to chronic pain treatment? Acta anaesthesiologica scandinavica 2001; 45(9):1128-35.

\section{Koltzenburg 2001}

Koltzenburg M, Scadding J. Neuropathic pain. Current opinion in neurology 2001; 14(5):641-7.

\section{Kurtzke 1983}

Kurtzke JF. Rating neurologic impairment in multiple sclerosis: an Expanded Disability Status Scale (EDSS). Neurology 1983; 33(11):1444-52.

\section{Langford 2013}

Langford RM, Mares J, Novotna A, Vachova M, Novakova I, Notcutt W, et al. A double-blind, randomized, placebocontrolled, parallel-group study of $\mathrm{THC} / \mathrm{CBD}$ oromucosal spray in combination with the existing treatment regimen, in the relief of central neuropathic pain in patients with multiple sclerosis. Journal of neurology 2013; 260(4):984-97.

\section{McDonald 2001}

McDonald WI, Compston A, Edan G, Goodkin D, , Lublin FD, et al. Recommended diagnostic criteria for multiple sclerosis: guidelines from the international panel on the diagnosis of multiple sclerosis. Annals of neurology 2001; 50(1):121-7.

\section{Melzack 1975}

Melzack R. The McGill Pain Questionnaire: major properties and scoring methods. Pain 1975; 1(3):277-99.

\section{Melzack 1987}

Melzack R. The short-form McGill Pain Questionnaire. Pain 1987; 30(2):191-7.

\section{Moore 2010}

Moore AR, Eccleston C, Derry S, Wiffen P, Bell RF, Straube S, et al. "Evidence" in chronic pain - establishing best practice in the reporting of systematic reviews. Pain 2010; 150(3):386-9.

\section{Motl 2010}

Motl RW, Suh Y, Weikert M. Symptom cluster and quality of life in multiple sclerosis. Journal of pain and symptom management 2010; 39(6):1025-32.

\section{O'Connor 2008}

O'Connor AB, Schwid SR, Herrmann DN, Markman JD, Dworkin RH. Pain associated with multiple sclerosis: systematic review and proposed classification. Pain 2008; 137(1):96-111.

\section{Osterberg 2005}

Osterberg A, Boivie J, Thuomas KA. Central pain in multiple sclerosis - prevalence and clinical characteristics. European journal of pain 2005; 9(5):531-42.

\section{Page 2019}

Page MJ, Higgins JP, Sterne JA. Chapter 13: Assessing risk of bias due to missing results. In: Higgins JP, Thomas J, Chandler J, Cumpston M, Li T, Page MJ, et al, editor(s). Cochrane Handbook for Systematic Reviews of Interventions version 6.0 (updated July 2019). Cochrane, 2019. Available from www.training.cochrane.org/handbook.

\section{Pasternak 2010}

Pasternak GW. Molecular insights into mu opioid pharmacology: from the clinic to the bench. Clinical journal of pain 2010; 26(Suppl 10):S3-9.

\section{Polman 2005}

Polman CH, Reingold SC, Edan G, Filippi M, , Kappos L, et al. Diagnostic criteria for multiple sclerosis: 2005 revisions to the "McDonald criteria". Annals of neurology 2005; 58(6):840-6.

\section{Polman 2011}

Polman CH, Reingold SC, Banwell B, Clanet M, Cohen JA, Filippi M, et al. Diagnostic criteria for multiple sclerosis: 2010 revisions to the McDonald criteria. Annals of neurology 2011; 69(2):292-302.

\section{Poser 1983}

Poser CM, Paty DW, Scheinberg L, McDonald WI, Davis FA, Ebers GC, et al. New diagnostic criteria for multiple sclerosis: guidelines for research protocols. Annals of neurology 1983; 13(3):227-31.

\section{Pöllmann 2008}

Pöllmann W, Feneberg W. Current management of pain associated with multiple sclerosis. CNS drugs 2008; 22(4):291-324.

\section{Review Manager 2014 [Computer program]}

Nordic Cochrane Centre, The Cochrane Collaboration Review Manager 5 (RevMan 5). Version 5.3. Copenhagen: Nordic Cochrane Centre, The Cochrane Collaboration, 2014. 


\section{Rog 2005}

Rog DJ, Nurmikko TJ, Friede T, Young CA. Randomized, controlled trial of cannabis-based medicine in central pain in multiple sclerosis. Neurology 2005; 65(6):812-9.

\section{Schünemann 2019}

Schünemann HJ, Higgins JP, Vist GE, Glasziou P, Akl EA, Skoetz N, et al. Chapter 14: Completing "Summary of findings" tables and grading the certainty of the evidence. In: Higgins JP, Thomas J, Chandler J, Cumpston M, Li T, Page MJ, et al, editor(s). Cochrane Handbook for Systematic Reviews of Interventions version 6.0 (updated July 2019). Cochrane, 2019. Available from www.training.cochrane.org/handbook.

\section{Shibasaki 1974}

Shibasaki $\mathrm{H}$, Kuroiwa Y. Painful tonic seizure in multiple sclerosis. Archives of neurology 1974; 30(1):47-51.

\section{Solaro 2013}

Solaro C, Trabucco E, Messmer Uccelli M. Pain and multiple sclerosis: pathophysiology and treatment. Current neurology and neuroscience reports 2013; 13(1):320.

\section{Stenager 1991}

Stenager E, Knudsen L, Jensen K. Acute and chronic pain syndrome in multiple sclerosis. Acta neurologica scandinavia 1991; 84(3):197-200

\section{Sterne 2011}

Sterne JA, Sutton AJ, loannidis JP, Terrin N, Jones DR, Lau J, et al. Recommendations for examining and interpreting funnel plot asymmetry in meta-analyses of randomised controlled trials. BMJ 2011; 343:d4002.

\section{Svendsen 2004}

Svendsen KB, Jensen TS, Bach FW. Does the cannabinoid dronabinol reduce central pain in multiple sclerosis? Randomised double blind placebo controlled crossover trial. BMJ 2004; 329(7460):253.

\section{Thompson 2018}

Thompson AJ, Banwell BL, Barkhof F, Carroll WM, Coetzee T, Comi G, et al. Diagnosis of multiple sclerosis: 2017 revisions of the McDonald criteria. Lancet neurology 2018; 17(2):162-73. [DOI: 10.1016/S1474-4422(17)30470-2] [PMID: 29275977]

\section{Treede 2008}

Treede RD, Jensen TS, Campbell JN, Cruccu G, Dostrovsky JO, Griffin JW, et al. Neuropathic pain: redefinition and a grading system for clinical and research purposes. Neurology 2008; 70(18):1630-5.

\section{Vickrey 1995}

Vickrey BG, Hays RD, Harooni R, Myers LW, Ellison GW. A healthrelated quality of life measure for multiple sclerosis. Quality of life research 1995; 4(3):187-206.

\section{Wang 2004}

Wang GK, Russell C, Wang SY. State-dependent block of voltagegated $\mathrm{Na}+$ channels by amitriptyline via local anesthetic receptor and its implication for neuropathic pain. Pain 2004; 110(1-2):166-74

\section{Ware 1992}

, Sherbourne CD. The MOS 36-item short-form health survey (SF-36). I. Conceptual framework and item selection. Medical care 1992; 30(6):473-83.

\section{Woolf 2000}

Woolf CJ, Salter MW. Neuronal plasticity: increasing the gain in pain. Science 2000; 288(5472):1765-9.

\section{Yamout 2013}

Yamout B, Issa Z, Herlopian A, El Bejjani M, Khalifa A, Ghadieh AS, et al. Predictors of quality of life among multiple sclerosis patients: a comprehensive analysis. European journal of neurology 2013; 20(5):756-64.

\section{A P P E N D I C E S}

\section{Appendix 1. Keywords for search strategy}

\{dysaesthesia \} OR \{dysesthesia \} OR \{dysaesthetic\} OR \{dysesthetic\} OR \{paresthesia\} OR \{pain\} OR \{central pain\} OR \{neuropathic pain\} OR \{chronic pain\} OR \{central neuropathic pain\} OR \{trigeminal neuralgia\} OR \{lhermitte's\} OR \{painful tonic spasm $\left.\backslash^{\star}\right\}$ OR $\{$ headache OR \{central neuropathic extremity pain\} AND \{tricyclics\} OR \{selective noradrenaline reuptake inhibitors\} OR \{opioids\} OR \{ $\alpha-2-\delta$ ligands\} OR \{gabapentin\} OR \{pregabalin\} OR \{sodium channel blockers\} OR \{antiepileptic\} OR \{anticonvulsants\} OR \{antidepressants\} OR \{antispasmodics\} OR \{opiates\} OR \{cannabinoids\} OR \{non steroidal anti-inflammatory drugs\} OR \{NSAIDS\} OR \{anaesthetic\} OR \{local anaesthetic\} OR \{membrane stabilising agents\} OR \{steroids\} OR \{capsaicin\}

\section{H I S T O R Y}

Protocol first published: Issue 4, 2020

\section{CONTRIBUTIONSOFAUTHORS}

EJ: read and approved the final version of the protocol.

CAY: drafted the protocol; read and approved the final version of the protocol.

EG: read and approved the final version of the protocol. 
AS: read and approved the final version of the protocol.

CT: drafted the protocol; read and approved the final version of the protocol.

\section{DECLARATIONS OF INTEREST}

This Cochrane Review has no commercial sponsorship.

EJ: none.

CAY: secured financial support from Janssen-Cilag Limited in 2001 to conduct a clinical trial using topiramate in people with multiple sclerosis and neuropathic pain. She has not been involved in a pain trial since then. CAY has had grants from pharmaceutical companies and has been an investigator in commercial trials, all funding held by the National Health Service. CAY has participated in educational meetings for MS and other diseases sponsored by large pharmaceutical companies, including receiving speakers fees. CAY has performed consultancy work unrelated to pain or its treatment, for Wellcome, Actelion, Biogen, Merck, Novartis, Roche, and Teva.

EG: none.

AS: none.

$\mathrm{CT}$ : participated in educational meetings for MS and other diseases sponsored by large pharmaceutical companies, including receiving speakers fees.

\section{SOURCES OF SUPPORT}

\section{Internal sources}

- No sources of support provided, Other

\section{External sources}

- No sources of support provided, Other 